\title{
Laparoscopic removal of gastrointestinal stromal tumors of uncinate process of pancreas
}

\author{
Maciej Stanek, Michał Pędziwiatr, Maciej Matłok, Andrzej Budzyński \\ $2^{\text {nd }}$ Department of Surgery, Jagiellonian University Medical College, Krakow, Poland
}

Videosurgery Miniinv 2015; 10 (2): 311-315

DOI: $10.5114 /$ wiitm.2015.52141

\begin{abstract}
The pancreas is an extremely rare location for gastrointestinal stromal tumors (GIST). We present a case of a patient with a GIST located in the uncinate process of the pancreas that was treated successfully with a laparoscopic technique. Computed tomography, magnetic resonance imaging and scintigraphy suggested a neuroendocrine tumor. Due to the fact that the image suggested a neuroendocrine tumor with a diameter below $2 \mathrm{~cm}$, the patient was qualified for a laparoscopic procedure of tumor enucleation. Postoperative care proceeded in accordance with the principles of the ERAS concept. The postoperative course was uncomplicated. He was discharged home on the second postoperative day. In the obtained histopathology result a GIST was found. During a 6-month observation, including control computed tomography examination, no signs of tumor progression were found. Despite the fact that stromal tumors of the gastrointestinal tract localized in the pancreas are very rare, they should be considered in the differential diagnosis of tumors of this organ.
\end{abstract}

Key words: laparoscopic surgery, gastrointestinal stromal tumor, pancreatic tumor.

\section{Introduction}

Gastrointestinal stromal tumors (GIST) are rare lesions comprising about $1 \%$ of neoplasms of the gastrointestinal tract [1]. They are usually localized in the stomach (60\%), but they can involve the small intestine (30\%), large intestine (4\%), or esophagus $(<1 \%)[2,3]$. Even rarer cases of GIST developing outside of the GI tract (extragastrointestinal stromal tumors - EGISTs), in organs such as the pancreas, gallbladder, urinary bladder, or omentum, are described [4]. The mainstay of treatment of GIST is surgery based on resection of the lesion with a healthy tissue margin [5].

Below we present a case of a patient with GIST located in the uncinate process of the pancreas that was treated successfully with a laparoscopic technique.

\section{Case report}

A 55-year-old man came to our clinic because of a $1.5 \mathrm{~cm}$ pancreatic tumor that was accidentally found during a computed tomography examination (Photo 1). At that time the patient was completely asymptomatic. In order to widen the diagnostics he was referred for magnetic resonance imaging study of the abdominal cavity. In that examination a lesion sized $20 \times 17 \times 16$ located in the uncinate process of the pancreas was found. It intensified significantly after intravenous contrast administration (Photo 2). The image primarily suggested a neuroendocrine tumor. To confirm the diagnosis, scintigraphy was performed using a marked somatostatin analogue (99MTC). The examination confirmed the presence of an area of higher expression of somatostatin receptors located in the uncinate process of the pancreas

\section{Address for correspondence}

Maciej Stanek, $2^{\text {nd }}$ Department of Surgery, Jagiellonian University Medical College, 21 Kopernika St, 31-501 Krakow, Poland,

phone: +48 698150 053, e-mail: maciej.m.stanek@gmail.com 


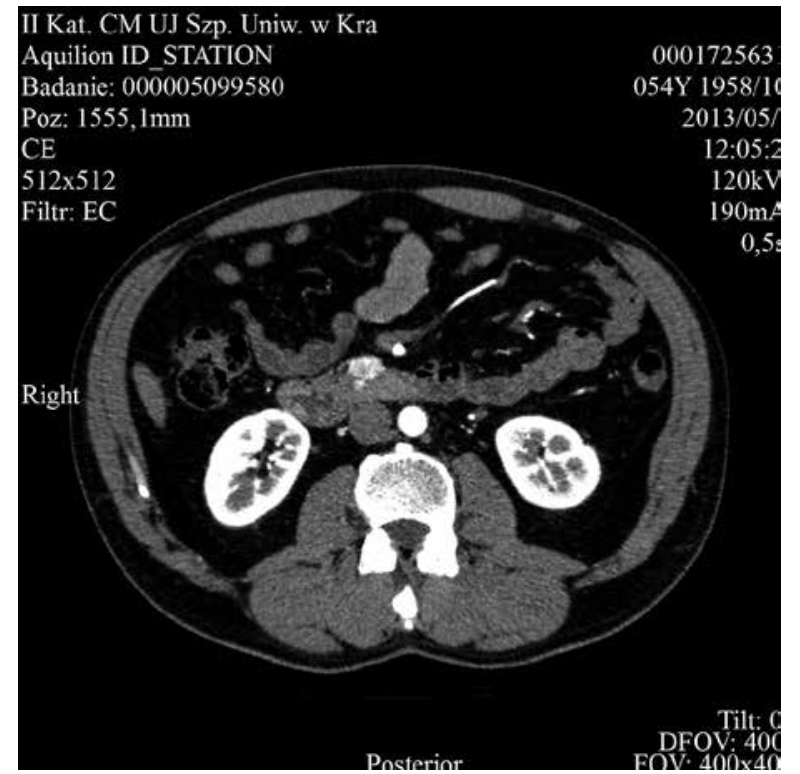

Photo 1. Computed tomography image showing a tumor in the area of the uncinate process of the pancreas

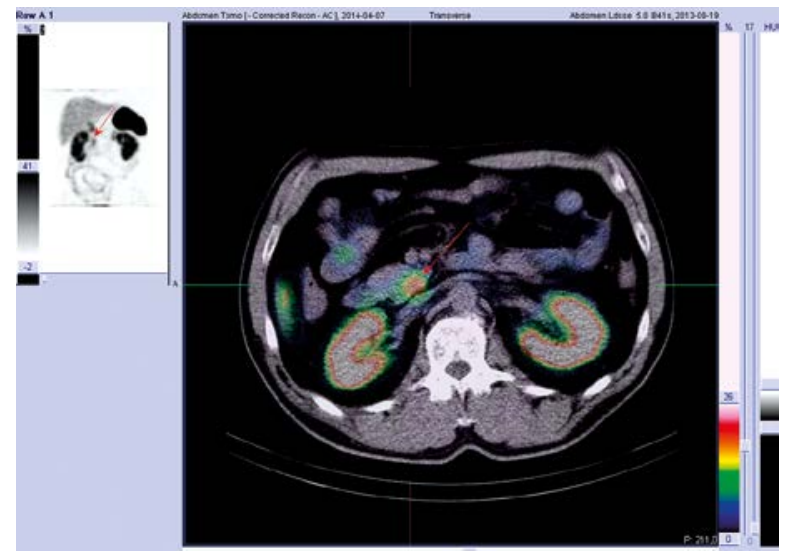

Photo 3. Scintigraphy with a marked somatostatin analogue (99MTC) confirming presence of an area of increased expression of somatostatin receptors localized in the uncinate process of the pancreas

(Photo 3). In the imaging studies no distal lesions were found within the abdominal cavity. No cancer markers were elevated either. Due to the fact that the image suggested a neuroendocrine tumor with a diameter below $2 \mathrm{~cm}$, the patient was qualified for a laparoscopic procedure of tumor enucleation. The procedure was performed using 5 ports (Figure 1). Intraoperative ultrasound was performed, visualizing a $15 \mathrm{~mm}$ hypoechogenic structure within the

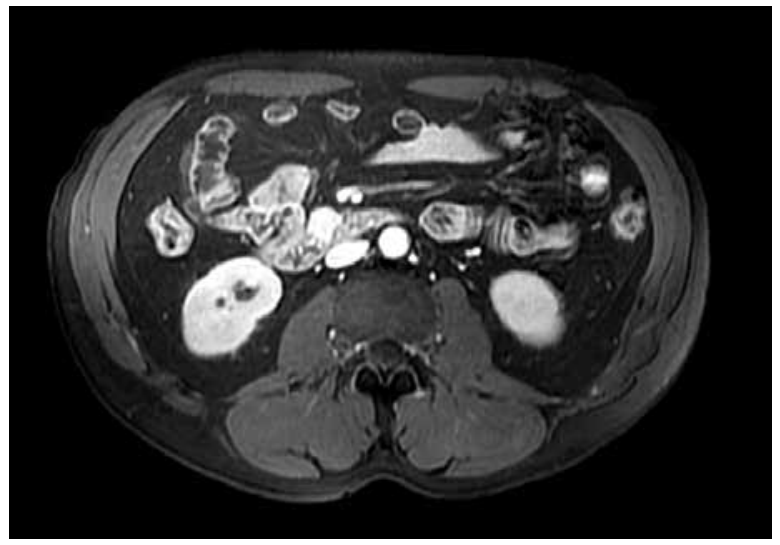

Photo 2. Magnetic resonance imaging image showing a lesion in the uncinate process of the pancreas sized $20 \times 17 \times 16 \mathrm{~mm}$, which significantly intensified after intravenous contrast administration

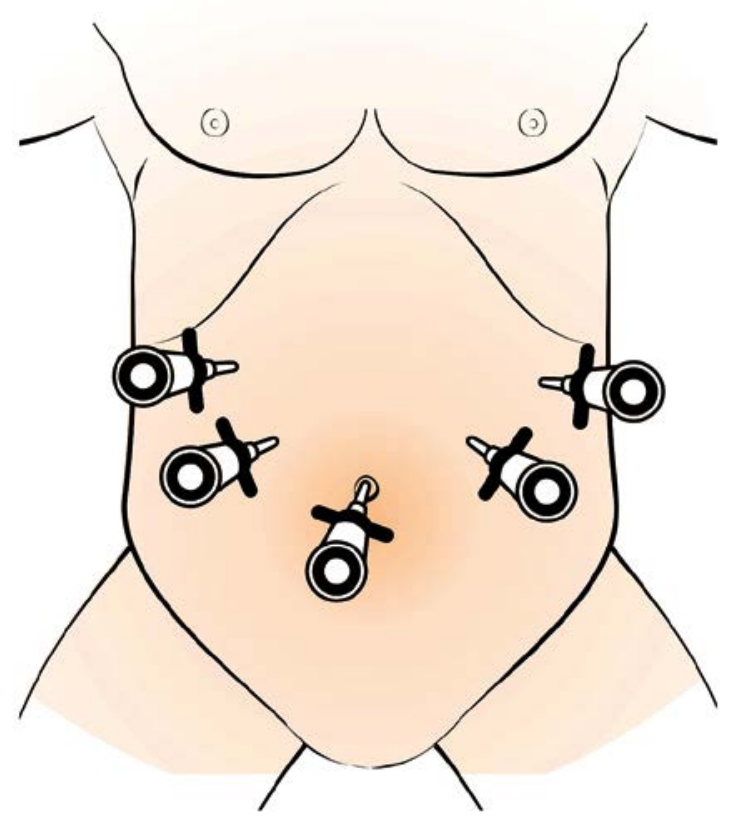

Figure 1. Trocar placement during the procedure

uncinate process of the pancreas, which was then enucleated completely and sent for histopathologic verification (Photos 4 and 5). No drain was left in the abdominal cavity. Postoperative care proceeded in accordance with the principles of the ERAS concept [6]. A few hours after the procedure the patient started to walk around. During the first $24 \mathrm{~h}$ drinks and a fluid diet were administered. The further postoperative course was uncomplicated. The patient 


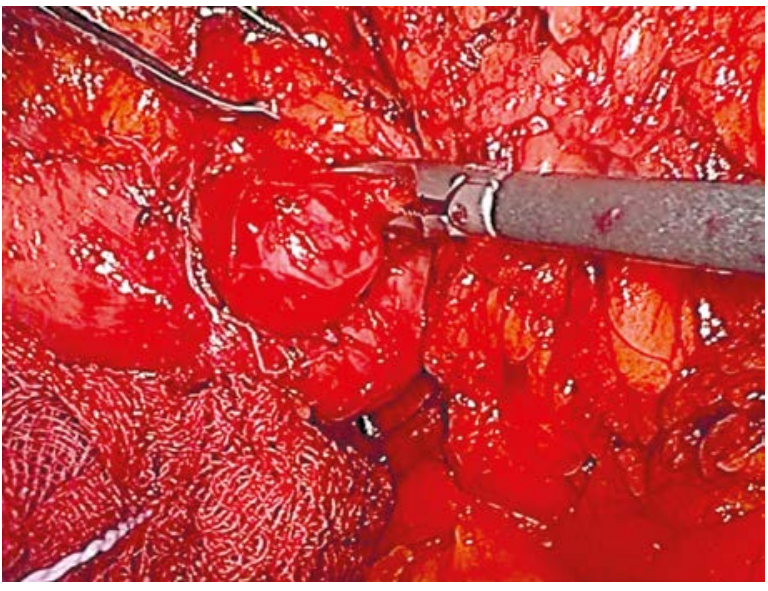

Photo 4. Intraoperative image of the tumor. Stage of tumor enucleation from the uncinate process of the pancreas

required use of small doses of nonsteroidal anti-inflammatory drugs (NSAIDs) due to moderately severe abdominal pain. He was discharged home on the second postoperative day. In the obtained histopathology result a GIST was found (Photos 6 and 7) with a diameter of $1.8 \mathrm{~cm}$ and 1 figure of mitotic division per 50 high power fields of view (Table I). The risk of tumor progression assessed according to the Joensuu criteria was defined as low. The histopathologic examination confirmed an RO resection. Because of this, it was decided not to widen the margin of excision, even though the resection margin was smaller than $10 \mathrm{~mm}$. During 6 months of observation, including control computed tomography examination, no signs of tumor progression were found.

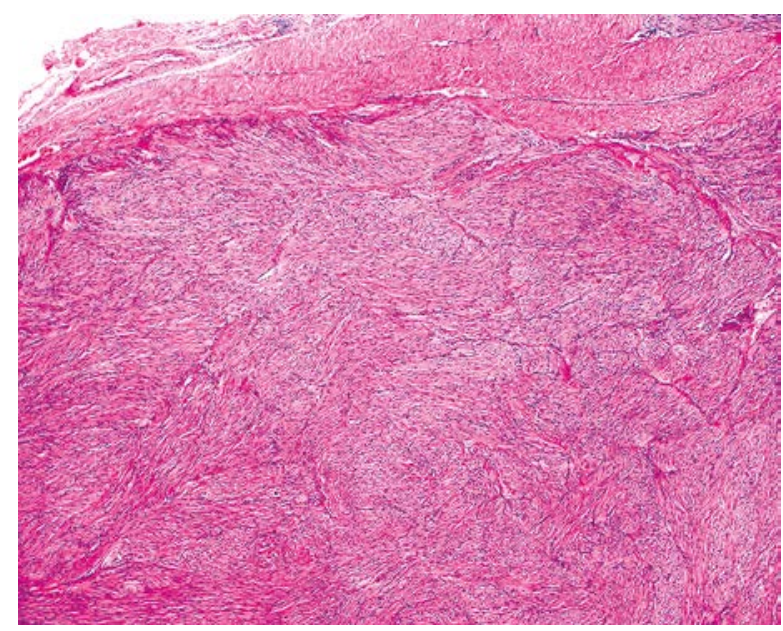

Photo 6. Microscopic image of the removed tumor. Fusiform GIST cells together with a neighboring strand of fibrous tissue

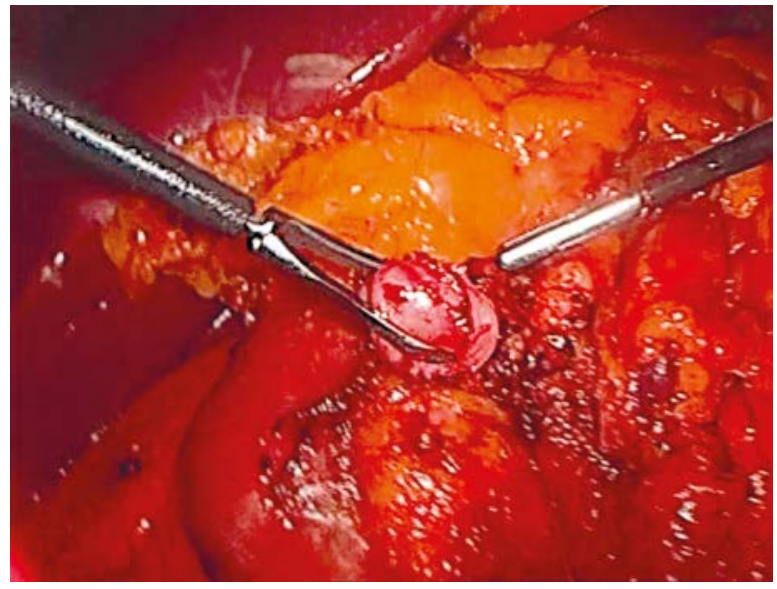

Photo 5. Intraoperative image of the tumor. Completely enucleated tumor

Table I. Pathological characteristics of the removed tumor

\begin{tabular}{|lc|}
\hline Tumor diameter & $18 \mathrm{~mm}$ \\
\hline Histological type & Fusiform \\
\hline Number of mitoses & $1 / 50 \mathrm{hpf}$ \\
\hline Immunohistochemical phenotype & $\begin{array}{c}\text { CD117 +, S100 +, } \\
\text { SMA + }\end{array}$ \\
\hline Risk of progression according to Joensuu & Very low \\
\hline
\end{tabular}

\section{Discussion}

The GIST are rare lesions, and their occurrence outside of the gastrointestinal tract (EGIST) is sporad-

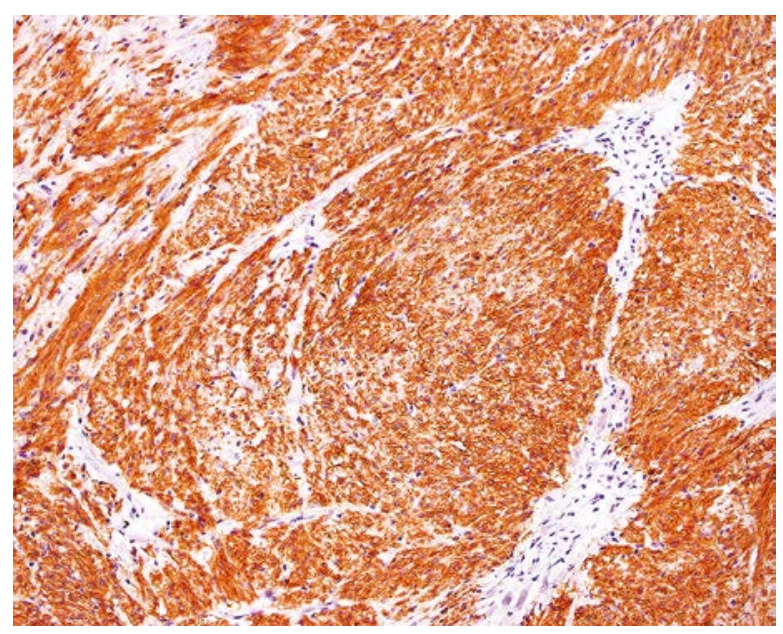

Photo 7. Microscopic image of the removed tumor. Positive immunohistochemical staining for CD 117 
ic. These neoplasms may also be located in the urinary bladder, omentum, gallbladder, or retroperitoneal space and pancreas, among other locations [4, 7].

Most pancreatic tumors are lesions of epithelial origin. Neuroendocrine tumors comprise a small percentage of lesions of this organ [8]. The pancreas is an extremely rare location for EGIST. So far about 20 cases of neoplasms of this type localized in the pancreas have been described [9].

The mainstay of GIST treatment is surgical treatment based on a radical excision of the lesion with a macroscopically healthy tissue border with a 1-2 cm margin. Unlike malignant tumors of epithelial origin, removal of the regional lymphatic system seems unnecessary, because neoplasms of the GIST type very rarely metastasize to lymph nodes [10]. Such characteristics encourage the use of minimally invasive techniques in treatment of these neoplasms [11]. Most cases of pancreatic EGIST described in the literature were large lesions [9]. In more than a half of them the tumor diameter was over $10 \mathrm{~cm}$, and the lesion itself often extended beyond the pancreas. Because of that, the surgical treatment involved extensive resection procedures with the classical method. However, in the case described by us the pancreatic tumor was small. We decided on enucleation of the lesion because the preoperative tests suggested presence of a NET type tumor with a diameter below $2 \mathrm{~cm}$. The small size of the lesion and lack of infiltration of neighboring tissues allowed the use of laparoscopy, even though the tumor was located in the uncinate process of the pancreas, which from the anatomical point of view seems to be a place that is hard to reach in surgery. The lesion was removed with a small healthy tissue margin, because its widening would be connected with the need of pancreaticoduodenectomy. According to our knowledge the procedure performed by us is the first surgery of that kind performed within the pancreas due to GIST.

Due to a small number of pancreatic GIST cases described in the literature we only have rudimentary information concerning the results of treatment of these tumors. However, existing scientific papers comparing laparoscopic treatment of stomach GIST with the procedures performed using the classic method suggest that the minimally invasive treatment allows similar results to be obtained in terms of oncology, and at the same time, it is linked with a smaller perioperative injury, less blood loss, and a quicker recovery of proper function of the gastrointestinal tract. It shortens the time of hospitalization and reduces the risk of complications [12]. Due to the very rare occurrence in the literature there is a lack of scientific research documenting the advantage of one method over the other in the case of GIST localized in the pancreas.

In the case described by us, perioperative procedures according to the ERAS concept were implemented [6]. Postoperative care based on the principles of, among others, early mobilization of the patient, early administration of per os feeding and optimal analgesia, allowed the patient to be discharged home within $48 \mathrm{~h}$ of the procedure.

Clinically, stromal tumors of the gastrointestinal tract are characterized by a high variability. They range from small lesions with a slow, almost stationary course, recognized often accidentally during endoscopic examinations or surgical procedures, to tumors with a very aggressive clinical picture with multiple metastases at the time of diagnosis [13]. Basic criteria allowing the determination of the degree of GIST malignancy include characteristics such as tumor size, value of the mitotic index, and lesion location [1, 14]. Available literature suggests that GIST located in the stomach are characterized by a better prognosis than GIST in other locations. The limited quantity of data does not allow us to clearly determine the prognosis in the case of pancreatic GIST. However, in accordance with the above information it can be suspected that GIST of the pancreas have a worse prognosis than lesions located in the stomach. This necessitates special oncological caution in future follow-ups. In the case presented by us, the tumor was characterized by a low degree of malignancy and was radically removed. However, the short follow-up period does not allow the assessment of long-term treatment.

\section{Conclusions}

Despite the fact that stromal tumors of the gastrointestinal tract localized in the pancreas are very rare, they should be considered in the differential diagnosis of tumors of this organ. The case described by us is an example showing that minimally invasive procedures can be used in the treatment of this type of tumor within the pancreas. Nevertheless, it is necessary for the operator to have significant experience and proficiency in surgical procedures due to oncological indications. Also, patients should remain 
under constant postoperative care, irrespective of the degree of tumor malignancy.

\section{Conflict of interest}

The authors declare no conflict of interest.

\section{References}

1. Miettinen M, Majidi M, Lasota J. Pathology and diagnostic criteria of gastrointestinal stromal tumors (GISTS): a review. Eur J Cancer 2002; 38 Suppl 5: S39-51.

2. Miettinen M, Lasota J. Gastrointestinal stromal tumors: pathology and prognosis at different sites. Semin Diagn Pathol 2006; 23: 70-83.

3. Poškus E, Petrik P, Petrik E, et al. Surgical management of gas trointestinal stromal tumors: a single center experience. Videosurgery Miniinv 2014; 9: 71-82.

4. Zhao X, Yue C. Gastrointestinal stromal tumor. J Gastrointest Oncol 2012; 3: 189-208.

5. Casali PG, Blay JY. Gastrointestinal stromal tumours: ESMO Clinical Practice Guidelines for diagnosis, treatment and follow-up. Ann Oncol 2010; 21 Suppl 5: v98-102.

6. Lassen K, Coolsen M, Slim K, et al. Guidelines for perioperative care for pancreaticoduodenectomy: Enhanced Recovery After Surgery $\left(\right.$ ERAS $\left.^{\circledR}\right)$ Society recommendations. Clin Nutr 2012; 31: 817-30.

7. Roggin K, Posner M. Modern treatment of gastric gastrointes tinal stromal tumors. World J Gastroenterol 2012; 18: 6720-8.

8. Rustagi T, Farrell JJ. Endoscopic diagnosis and treatment of pancreatic neuroendocrine tumors. J Clin Gastroenterol 2014; 48 837-44.

9. Padhi S, Sarangi R, Mallick S. Pancreatic extragastrointestinal stromal tumors, interstitial Cajal like cells, and telocytes. JOP 2013; 14: 1-14.

10. Sicklick JK, Lopez NE. Optimizing surgical and imatinib therapy for the treatment of gastrointestinal stromal tumors. J Gastrointest Surg 2013; 17: 1997-2006.

11. Sákra L, Siller J, Vyhnálek P, et al. Surgical treatment of gastric and small bowel gastrointestinal stromal tumours. Videosurgery Miniinv 2011; 6: 138-43.

12. Liang JW, Zheng ZC, Zhang JJ, et al. Laparoscopic versus open gastric resections for gastric gastrointestinal stromal tumors: a meta-analysis. Surg Laparosc Endosc Percutan Tech 2013; 23: 378-87.

13. Al-Kalaawy M, El-Zohairy MA, Mostafa A, et al. Gastrointestinal stromal tumors (GISTs), 10-year experience: patterns of failure and prognostic factors for survival of 127 patients. J Egypt Natl Canc Inst 2012; 24: 31-9.

14. Joensuu $H$, Vehtari A, Riihimäki J, et al. Risk of recurrence of gastrointestinal stromal tumour after surgery: an analysis of pooled population-based cohorts. Lancet Oncol 2012; 13: 265-74.

Received: 18.01.2015, accepted: 16.03.2015. 